\title{
17. COMPRESSIONAL AND SHEAR WAVE VELOCITIES AND ELASTIC MODULI OF BASALTS, DEEP SEA DRILLING PROJECT, LEG 19
}

\author{
Nikolas I. Christensen, University of Washington, Seattle, Washington
}

\section{INTRODUCTION}

Geophysical interpretation depends upon a knowledge of the physical properties of rocks and rock forming minerals. The structure of the oceanic crust has been defined principally from seismic refraction investigations and interpretations of these studies require detailed information on seismic velocities in oceanic rocks. In this paper compressional and shear wave velocities are reported as a function of hydrostatic pressure for five specimens of basalt from three sites of Leg 19 of the Deep Sea Drilling Project.

\section{RESULTS}

Compressional and shear wave velocities at pressures to $10 \mathrm{~kb}$ and bulk densities calculated from the weights and dimensions of the samples are given in Table 1. All velocities were measured at room temperature from watersaturated cylindrical specimens $1.3 \mathrm{~cm}$ in diameter and 3 to $4 \mathrm{~cm}$ in length. Velocities were measured in two perpendicular cores for the basalts from Sites 191 and 192. The laboratory techniques employed have been described in detail by Birch (1960) and Christensen and Shaw (1970).

The ratio of compressional to shear velocity $\left(\mathrm{V}_{\mathrm{p}} / \mathrm{V}_{\mathrm{s}}\right)$, Poisson's ratio $(\sigma)$, the seismic parameter $(\phi)$, the bulk modulus $(\mathrm{K})$, compressibility $(\beta)$, the shear modulus $(\mu)$, Young's modulus (E) and Lame's constant $(\lambda)$ calculated from mean velocities and densities corrected for dimension changes (Christensen and Shaw, 1970) are presented in Table 2. Petrographic descriptions of the samples included in this study are given by Stewart et al. elsewhere in this volume.

\section{DISCUSSION}

The commonly observed rapid increase of velocity with increasing pressure to approximately $2 \mathrm{~kb}$ is a consequence of the closure of grain boundary cracks and the accompanying reduction in porosity (Birch, 1961). Compressional wave velocities at relatively low pressures are significantly higher if the rock pore spaces are water saturated (Dortman and Magid, 1968; Nur and Simmons, 1969; Christensen, 1970). This is illustrated in Figure 1 where compressional wave velocities from a saturated specimen from Site 192 (pore pressure $<<$ external pressure) are compared with velocities measured from the same core after it was air dried for 24 hours at room temperature. The pressures at which most basalts are recovered from the Deep Sea Drilling Project are usually between 0.4 and $0.6 \mathrm{~kb}$. At these pressures, compressional wave velocities in dry samples are usually 10 percent lower than measurements made on water saturated samples. It is thus extremely important when comparing laboratory measurements of basalt compressional wave velocities with in situ velocities to use velocities from water-saturated specimens. Shear wave velocities, on the other hand, are little affected by water saturation.

Both compressional and shear wave velocities of the basalts show systematic decreases with decreasing density. This is illustrated in Figures 2 and 3 where the velocities and densities measured for the Leg 19 basalts are compared with linear least-squares solutions of velocity and density for fourteen specimens of basalt from Legs 2, 3, and 4 in the Atlantic Ocean (Christensen and Salisbury, 1972). For a given density there appear to be no significant differences

TABLE 1

Compressional (P) and Shear (S) Wave Velocities

\begin{tabular}{|c|c|c|c|c|c|c|c|c|c|c|c|c|}
\hline \multirow[b]{2}{*}{ Sample } & \multirow{2}{*}{$\begin{array}{c}\text { Bulk } \\
\text { Density }\end{array}$} & \multirow[b]{2}{*}{ Mode } & \multicolumn{10}{|c|}{ Velocity $(\mathrm{km} / \mathrm{sec})$ at Varying Pressures } \\
\hline & & & $0.2 \mathrm{~kb}$ & $0.4 \mathrm{~kb}$ & $0.6 \mathrm{~kb}$ & $0.8 \mathrm{~kb}$ & $1.0 \mathrm{~kb}$ & $2.0 \mathrm{~kb}$ & $4.0 \mathrm{~kb}$ & $6.0 \mathrm{~kb}$ & $8.0 \mathrm{~kb}$ & $10.0 \mathrm{~kb}$ \\
\hline \multirow{6}{*}{$\begin{array}{l}19-192 \mathrm{~A}-5-4 \\
(133-142 \mathrm{~cm})\end{array}$} & 2.570 & $P$ & 4.81 & 4.87 & 4.92 & 4.95 & 4.982 & 5.095 & 5.249 & 5.359 & 5.446 & 5.529 \\
\hline & 2.555 & P & 4.72 & 4.77 & 4.83 & 4.88 & 4.922 & 5.082 & 5.276 & 5.408 & 5.508 & 5.578 \\
\hline & $2.563^{\mathrm{a}}$ & $P$ & $4.77^{a}$ & $4.82^{\mathrm{a}}$ & $4.88^{\mathrm{a}}$ & $4.92^{\mathrm{a}}$ & $4.952^{\mathrm{a}}$ & $5.089^{\mathrm{a}}$ & $5.263^{a}$ & $5.384^{\mathrm{a}}$ & $5.477^{\mathrm{a}}$ & $5.554^{\mathrm{a}}$ \\
\hline & 2.570 & S & 2.44 & 2.46 & 2.48 & 2.49 & 2.511 & 2.577 & 2.661 & 2.703 & 2.733 & 2.750 \\
\hline & 2.555 & $\mathrm{~S}$ & 2.43 & 2.45 & 2.47 & 2.48 & 2.501 & 2.567 & 2.652 & 2.701 & 2.738 & 2.760 \\
\hline & $2.563^{a}$ & S & $2.44^{\mathrm{a}}$ & $2.46^{\mathrm{a}}$ & $2.48^{a}$ & $2.49^{\mathrm{a}}$ & $2.506^{\mathrm{a}}$ & $2.572^{\mathrm{a}}$ & $2.657^{\mathrm{a}}$ & $2.702^{\mathrm{a}}$ & $2.736^{\mathrm{a}}$ & $2.755^{\mathrm{a}}$ \\
\hline \multirow{6}{*}{$\begin{array}{l}19-191-16-1 \\
(21-25 \mathrm{~cm})\end{array}$} & 2.825 & $P$ & 5.61 & 5.64 & 5.66 & 5.68 & 5.690 & 5.743 & 5.812 & 5.867 & 5.923 & 5.970 \\
\hline & 2.762 & P & 5.42 & 5.44 & 5.46 & 5.47 & 5.483 & 5.532 & 5.595 & 5.647 & 5.697 & 5.740 \\
\hline & $2.794^{a}$ & $P$ & $5.52^{\mathrm{a}}$ & $5.54^{\mathrm{a}}$ & $5.56^{\mathrm{a}}$ & $5.58^{\mathrm{a}}$ & $5.587^{\mathrm{a}}$ & $5.638^{a}$ & $5.704^{a}$ & $5.757^{a}$ & $5.810^{\mathrm{a}}$ & $5.855^{\mathrm{a}}$ \\
\hline & 2.825 & S & 3.07 & 3.08 & 3.09 & 3.10 & 3.108 & 3.135 & 3.160 & 3.177 & 3.193 & 3.203 \\
\hline & 2.762 & S & 2.94 & 2.95 & 2.96 & 2.97 & 2.980 & 3.010 & 3.029 & 3.036 & 3.042 & 3.049 \\
\hline & $2.794^{a}$ & S & $3.01^{\mathrm{a}}$ & $3.02^{\mathrm{a}}$ & $3.03^{a}$ & $3.04^{\mathrm{a}}$ & $3.044^{\mathrm{a}}$ & $3.073^{\mathrm{a}}$ & $3.095^{\mathrm{a}}$ & $3.107^{a}$ & $3.118^{a}$ & $3.126^{\mathrm{a}}$ \\
\hline $19-183-39-1$ & 2.840 & $P$ & 6.14 & 6.16 & 6.18 & 6.20 & 6.218 & 6.281 & 6.357 & 6.405 & 6.427 & 6.434 \\
\hline$(148-150 \mathrm{~cm})$ & 2.840 & S & 3.08 & 3.09 & 3.11 & 3.12 & 3.131 & 3.182 & 3.240 & 3.263 & 3.272 & 3.273 \\
\hline
\end{tabular}

${ }^{a}$ Mean value. 
TABLE 2

Elastic Constants, Leg 19 Basalts

\begin{tabular}{cccccccccc}
\hline Site & $\begin{array}{c}\text { Pressure } \\
(\mathrm{kb})\end{array}$ & $\mathrm{V}_{\mathrm{p}} / \mathrm{V}_{\mathrm{S}}$ & $\sigma$ & $\begin{array}{c}\phi \\
(\mathrm{km} / \mathrm{sec})^{2}\end{array}$ & $\begin{array}{c}\mathrm{K} \\
(\mathrm{Mb})\end{array}$ & $\begin{array}{c}\beta \\
(\mathrm{Mb}-1)\end{array}$ & $\begin{array}{c}\mu \\
(\mathrm{Mb})\end{array}$ & $\begin{array}{c}\mathrm{E} \\
(\mathrm{Mb})\end{array}$ & $\begin{array}{c}\lambda \\
(\mathrm{Mb})\end{array}$ \\
\hline 183 & 0.4 & 1.99 & 0.33 & 25.2 & 0.72 & 1.40 & 0.27 & 0.72 & 0.53 \\
& 1.0 & 1.99 & 0.33 & 25.6 & 0.73 & 1.38 & 0.28 & 0.74 & 0.54 \\
& 2.0 & 1.97 & 0.33 & 25.9 & 0.74 & 1.36 & 0.29 & 0.76 & 0.55 \\
& 6.0 & 1.97 & 0.33 & 26.7 & 0.76 & 1.31 & 0.30 & 0.80 & 0.56 \\
& 10.0 & 1.97 & 0.33 & 26.9 & 0.77 & 1.29 & 0.31 & 0.81 & 0.60 \\
& 0.4 & 1.84 & 0.29 & 18.5 & 0.52 & 1.93 & 0.25 & 0.66 & 0.35 \\
& 1.0 & 1.84 & 0.29 & 18.8 & 0.53 & 1.90 & 0.26 & 0.67 & 0.35 \\
& 2.0 & 1.84 & 0.29 & 19.1 & 0.54 & 1.86 & 0.26 & 0.68 & 0.36 \\
& 6.0 & 1.85 & 0.29 & 20.1 & 0.57 & 1.76 & 0.27 & 0.70 & 0.39 \\
& 10.0 & 1.87 & 0.30 & 21.0 & 0.60 & 1.67 & 0.27 & 0.71 & 0.41 \\
& 0.4 & 1.96 & 0.32 & 15.2 & 0.39 & 2.57 & 0.16 & 0.41 & 0.28 \\
& 1.0 & 1.97 & 0.33 & 16.1 & 0.41 & 2.41 & 0.16 & 0.43 & 0.31 \\
& 2.0 & 1.98 & 0.33 & 17.0 & 0.44 & 2.28 & 0.17 & 0.45 & 0.33 \\
& 6.0 & 1.99 & 0.33 & 19.1 & 0.50 & 2.02 & 0.19 & 0.50 & 0.37 \\
& 10.0 & 2.02 & 0.34 & 20.5 & 0.53 & 1.87 & 0.20 & 0.52 & 0.40 \\
\hline
\end{tabular}

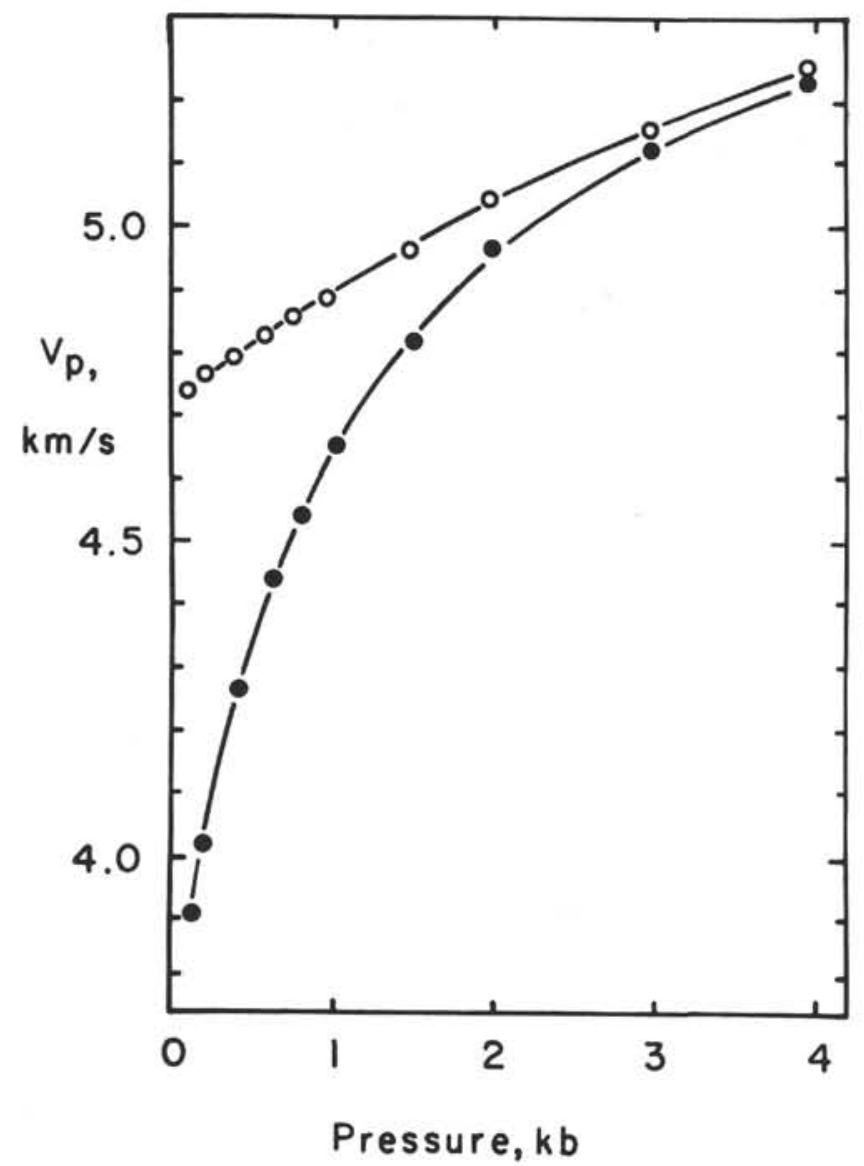

Figure 1. Velocities for a basalt from Site 192, water saturated (open circles) and air dry (solid circles).

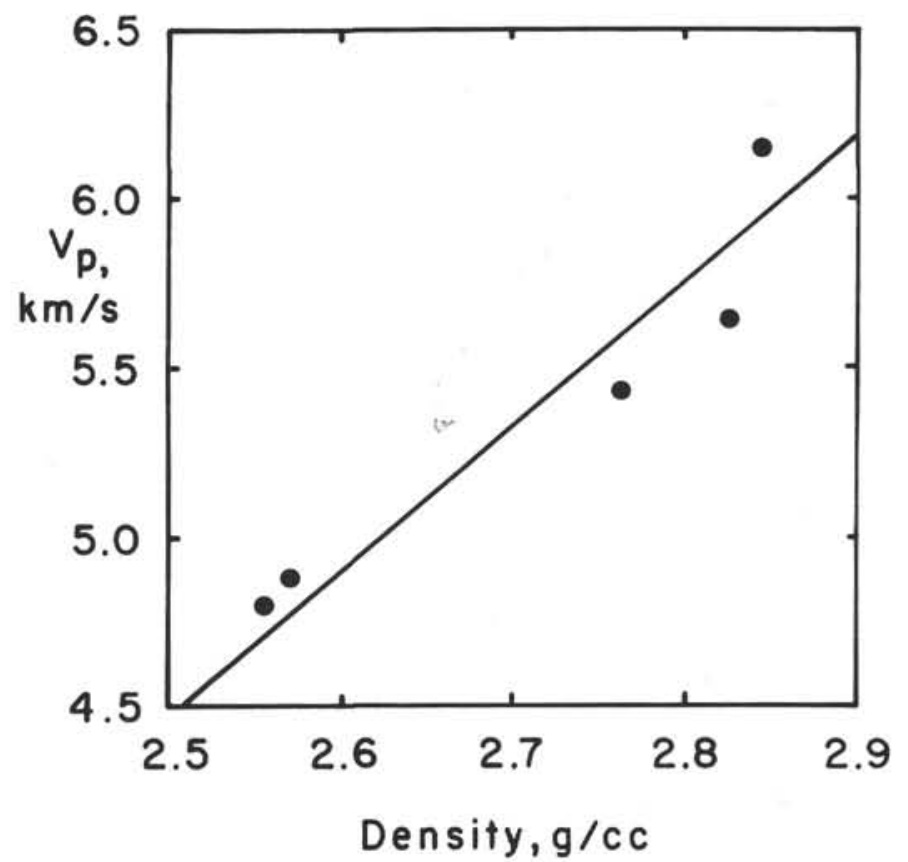

Figure 2. Compressional wave velocities versus densities at $0.5 \mathrm{~kb}$ (see text). 


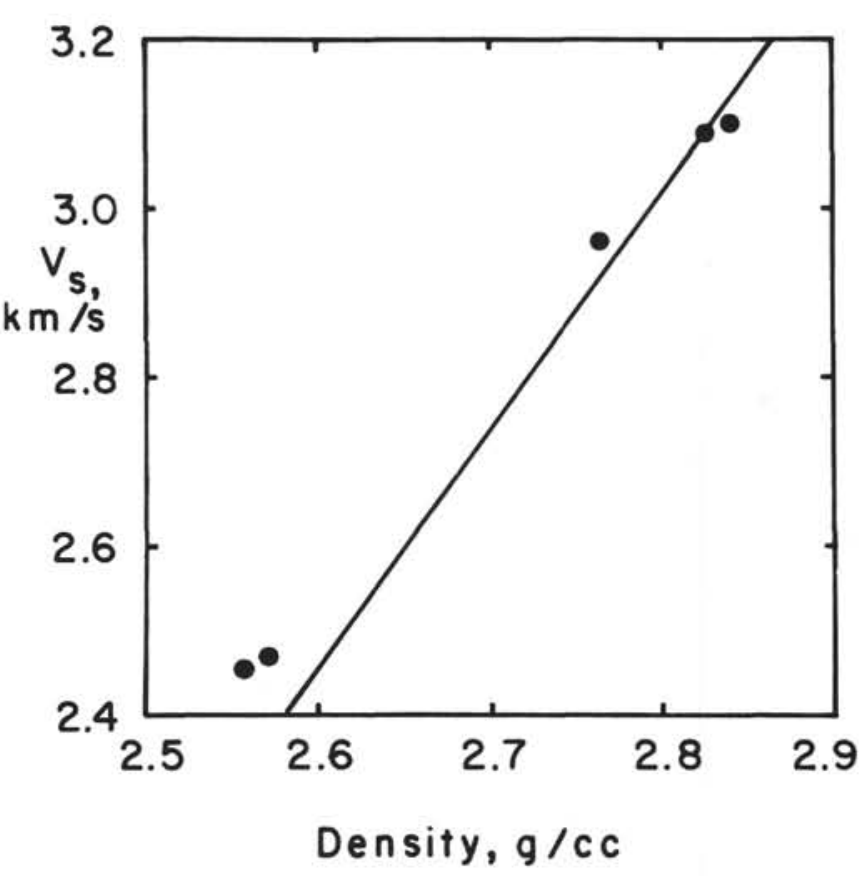

Figure 3. Shear wave velocities versus densities at $0.5 \mathrm{~kb}$ (see text). in the elastic properties of the basalts from Leg 19 and Legs 2,3 , and 4 . As was observed for the Atlantic basalts, the lower velocities and densities are a result of alteration.

\section{REFERENCES}

Birch, F., 1960. The velocity of compressional waves in rocks to 10 kilobars, 1. J. Geophys. Res. 65, 1083.

Birch, F., 1961. The velocity of compressional waves in rocks to 10 kilobars, 2. J. Geophys. Res. 66, 2199.

Christensen, N. I., 1970. Compressional wave velocities in basalts from the Juan de Fuca Ridge. J. Geophys. Res. $75,2773$.

Christensen, N. I. and Salisbury, M. H., in press. Sea floor spreading, progressive alteration of layer 2 basalts, and associated changes in seismic velocities. Earth Planet. Sci. Letters.

Christensen, N. I. and Shaw, G. H., 1970. Elasticity of mafic rocks from the Mid-Atlantic Rodge. Geophys. J. R. astr. Soc. 20, 271.

Dortman, N. B. and Magid, H. Sh., 1968. Velocity of elastic waves in crystalline rocks and its dependence on moisture content. Doklad Acad. Scien. USSR Earth Sci. Section 179,1 .

Nur, A. and Simmons, G., 1969. The effect of saturation on velocity in low porosity rocks. Earth Planet. Sci. Letters 7,183 . 